Journal of Universal Mathematics

Vol.3 No.1 PP.28-32 (2020)

ISSN-2618-5660

\title{
WEAK FINITELY \\ DUAL QUASI-CONJUGATIVE RELATIONS
}

\author{
DANIEL A. ROMANO
}

\begin{abstract}
The concept of 'weak finitely regular relations' was introduced by Shuzhen Luo and Xiaoquan Xu in 2019 and applied to partially ordered sets. In this article, this idea is extended to weak finality of dually quasi-conjugative relations was introduced in 2013 by this author.
\end{abstract}

\section{Introduction and Preliminaries}

For a set $X$, we call $\alpha$ a binary relation on $\mathrm{X}$ if $\alpha \subseteq X^{2}$. Let $\mathcal{B}(X)$ be denote the set of all binary relations on $\mathrm{X}$. For $\alpha, \beta \in \mathcal{B}(X)$, define

$$
\beta \circ \alpha=\left\{(x, z) \in X^{2}:(\exists y \in X)((x, y) \in \alpha,(y, z) \in \beta)\right\} .
$$

The relation $\beta \circ \alpha$ is called the composition of $\alpha$ and $\beta$. It is well known that $\mathcal{B}(X)$, with composition, is a monoid (semigroup with identity). Namely, $\triangle_{X}=$ $\{(x, x): x \in X\}$ is its identity element. For a binary relation $\alpha$ on a set $\mathrm{X}$, define $\alpha^{-1}=\left\{(x, y) \in X^{2}:(y, x) \in \alpha\right\}$ and $\alpha^{c}=X^{2} \backslash \alpha$. Thus $\left(\alpha^{c}\right)^{-1}=\left(\alpha^{-1}\right)^{c}$ holds.

Let $A$ be a subset of $X$. For $\alpha \in \mathcal{B}(X)$, set

$$
A \alpha=\{y \in X:(\exists a \in A)((a, y) \in \alpha)\}, \alpha A=\{x \in X:(\exists b \in A)((x, b) \in \alpha)\} .
$$

It is easy to see that $A \alpha=\alpha^{-1} A$ holds. Specially, we put $a \alpha$ instead of $\{a\} \alpha$ and $\alpha b$ instead of $\alpha\{b\}$.

Notions and notations used in this article that are not previously defined may be find by the reader in articles $[3,4,5,7,10]$.

1.1. Quasi-conjugative relations. The fundamental works of K. A. Zareckii [9], B. M. Schein [7] and others on regular relations motivated several mathematicians to investigate similar classes of relations, obtained by putting $\alpha^{-1}, \alpha^{c}$ or $\left(\alpha^{c}\right)^{-1}$ in place of one or both $\alpha$ 's on the right side of the regularity equation

$$
\alpha=\alpha \circ \beta \circ \alpha
$$

(where $\beta$ is some relation). The following class of elements in the semigroup $\mathcal{B}(X)$ have been investigated: normal relation in [1] by G. Jiang, L. Xu, J. Cai and G. Han; dually normal relation in [2] by G. Jiang and L. Xu; quasi-conjugative and bi-normal relations $[4,5,6,8]$ by this author and M. Vinčić. For example:

2000 Mathematics Subject Classification. 03E02; 06A11; $20 \mathrm{M} 20$.

Key words and phrases. quasi-conjugative relations, finitely dually quasi-conjugative relations, weak finitely dually quasi-conjugative relations. 
Definition 1.1. ([4], Definition 2.2) The relation $\alpha \in \mathcal{B}(X)$ is called a quasiconjugative if there exists a relation $\beta \in \mathcal{B}(X)$ such that

$$
\alpha=\alpha^{-1} \circ \beta \circ \alpha^{c} .
$$

The class of duals of these relations are given in the following definition.

Definition 1.2. ([4], Remark 2.1) The relation $\alpha \in \mathcal{B}(X)$ is called a dually quasiconjugative if there exists a relation $\beta \in \mathcal{B}(X)$ such that

$$
\alpha=\alpha^{c} \circ \beta \circ \alpha^{-1} \text {. }
$$

In the following propositions we give an intrinsic characterization of any quasiconjugative relation and its dual.

Proposition 1 ([4], Theorem 2.1). For a binary relation $\alpha$ on a set $X$, the following conditions are equivalent:

(1) $\alpha$ is a quasi-conjugative relation; and

(2) For all $x, y \in X$, if $(x, y) \in \alpha$, there exist $u, v \in X$ such that:

(a) $(x, v) \in \alpha^{c} \wedge(y, u) \in \alpha$;

(b) $\left.(\forall s, t \in X)\left((s, v) \in \alpha^{c} \wedge(t, u) \in \alpha\right) \Longrightarrow(s, t) \in \alpha\right)$.

Proposition 2. For a binary relation $\alpha$ on a set $X$, the following conditions are equivalent:

(1) $\alpha$ is a dually quasi-conjugative relation; and

(2) For all $x, y \in X$, if $(x, y) \in \alpha$, there exist $u, v \in X$ such that:

(a) $(x, v) \in \alpha^{-1} \wedge(u, y) \in \alpha^{c}$;

(b) $\left.(\forall s, t \in X)\left((s, v) \in \alpha^{-1} \wedge(u, t) \in \alpha^{c}\right) \Longrightarrow(s, t) \in \alpha\right)$.

1.2. Finitely quasi-conjugative relations. For any set $X$, let $X^{(<\omega)}=\{F \subseteq$ $X: F$ is finite $\}$. For any positive integer $m$, we write $\bar{m}=\{1,2, \ldots, m\}$.

Definition 1.3. ([10]) For a binary relation $\rho \subseteq X \times Y$, define a relation $\rho^{(<\omega)} \subseteq$ $X^{(<\omega)} \times Y^{(<\omega)}$ by

$$
\left(\forall(F, G) \in X^{(<\omega)} \times Y^{(<\omega)}\right)\left((F, G) \in \rho^{(<\omega)} \Longleftrightarrow G \subseteq F \rho\right) .
$$

$\rho^{(<\omega)}$ is called finite extension of $\rho$.

For illustration purposes, we will show definition of finite extension of quasiconjugative and dual quasi-conjugative relations:

Definition 1.4. ([5]) A binary relation $\alpha$ on a set $X$ is called finitely quasiconjugative if for all $(x, y) \in \alpha$, there are $u \in X$ and $\left\{v_{1}, v_{2}, \ldots, v_{k}\right\} \in X^{(<\omega)}$, such that

(i) $(u, y) \in \alpha^{-1} \wedge(\forall i \in \bar{k})\left(\left(x, v_{i}\right) \in \alpha^{c}\right)$, and

(ii) for all $\left\{s_{1}, s_{2}, \ldots, s_{k}\right\} \in X^{(<\omega)}$ and $t \in X$, if $(u, t) \in \alpha^{--1}$ and $(\forall i \in \bar{k})\left(\left(s_{i}, v_{i}\right) \in \alpha^{c}\right)$ then there is $j \in \bar{k}$ such that $\left(s_{j}, t\right) \in \alpha$.

An important description of the finitely bi-conjugative relation is given in the following proposition

Proposition $3([5])$. For a binary relation $\alpha$ on a set $X$, the following are equivalent:

(i) $\rho$ is a finitely quasi-conjugative relation on $X$; and

(ii) there is a binary relation $\delta \subseteq X^{(<\omega)} \times X^{(<\omega)}$ such that $\left[\left(\alpha^{--1}\right)^{(<\omega)} \circ \delta \circ\right.$ $\left.\left(\alpha^{c}\right)^{(<\omega)}=\alpha^{(<\omega)}\right]$ 
Definition 1.5. ([5]) A binary relation $\alpha$ on a set $X$ is called finitely dual quasiconjugative if for all $(x, y) \in \alpha$, there are $u \in X$ and $\left\{v_{1}, v_{2}, \ldots, v_{k}\right\} \in X^{(<\omega)}$, such that

(i) $(u, y) \in \alpha^{c} \wedge(\forall i \in \bar{k})\left(\left(x, v_{i}\right) \in \alpha^{-1}\right)$, and

(ii) for all $\left\{s_{1}, s_{2}, \ldots, s_{k}\right\} \in X^{(<\omega)}$ and $t \in X$, if $(u, t) \in \alpha^{c}$ and $(\forall i \in \bar{k})\left(\left(s_{i}, v_{i}\right) \in \alpha^{-1}\right)$ then there is $j \in \bar{k}$ such that $\left(s_{j}, t\right) \in \alpha$.

An important description of the finitely dual quasi-conjugative relation is given in the following proposition

Proposition $4([5])$. For a binary relation $\alpha$ on a set $X$, the following are equivalent:

(i) $\rho$ is a finitely dual quasi-conjugative relation on $X$; and

(ii) there is a binary relation $\delta \subseteq X^{(<\omega)} \times X^{(<\omega)}$ such that

$$
\left(\alpha^{c}\right)^{(<\omega)} \circ \delta \circ\left(\alpha^{-1}\right)^{(<\omega)}=\alpha^{(<\omega)} .
$$

\section{WEAK FINITELY DUAL QUASI-CONJUGATIVE RELATIONS}

In Definition 1.4 and 1.5, in statement (ii) the finite set $\left\{s_{1}, s_{2}, \ldots, s_{k}\right\},(k \in \mathbb{N})$ appears as a very strong condition. In the just published article [3], in a situation where regular relations is discussed, this requirement is weakened by taking $\{s\}$ instead of $\left\{s_{1}, s_{2}, \ldots, s_{k}\right\}$. We impose an analogous requirement on the finality of dually quasi-conjugative relations.

Before that, we need the following definition

Definition 2.1. ([3], Definition 6) For a binary relation $\rho \subseteq X \times Y$, define a binary relation $\rho^{(<\omega)} \subseteq X \times Y^{(<\omega)}$, called the right finite extension of $\rho$, by

$$
\rho_{r}^{(<\omega)}=\left\{(x, G) \in X \times Y^{(<\omega)}: G \subseteq x \rho\right\} .
$$

2.1. The concept. We can now introduce the concept of weak finitely dual quasiconjugative relations

Definition 2.2. A binary relation $\rho$ on a set $X$ is called weak finitely dual quasiconjugative if for all $(x, y) \in \rho$, there are $u \in X$ and $\left\{v_{1}, v_{2}, \ldots, v_{k}\right\} \in X^{(<\omega)}$, such that

(i) $(u, y) \in \rho^{c} \wedge(\forall i \in \bar{k})\left(\left(x, v_{i}\right) \in \rho^{-1}\right)$, and

(ii) for all $s \in X$ and $t \in X$,

if $(u, t) \in \rho^{c}$ and $(\forall i \in \bar{k})\left(\left(s, v_{i}\right) \in \rho^{-1}\right)$ then $(s, t) \in \rho$.

2.2. The main result. In the following theorem, we give some characterizations of weak finality of dually quasi-conjugative relations.

Theorem 2.3. Let $\rho \subseteq X \times X$ a binary relation. Then the following conditions are equivalent:

(1) $\rho$ is weak finitely dual quasi-conjugative;

(2) there is a relation $\delta \subseteq X^{(<\omega)} \times X^{(<\omega)}$ such that

$$
\left(\rho^{c}\right)^{(<\omega)} \circ \delta \circ\left(\rho_{r}^{-1}\right)^{(<\omega)}=\rho_{r}^{(<\omega)} ;
$$

(3) for all $(x, G) \in \rho_{r}^{(<\omega)}$ there is $(U, V) \in X^{(<\omega)} \times X^{(<\omega)}$ such that

(i) $G \subseteq U \rho^{c} \wedge V \subseteq \rho x$, and

(ii) for any $(s, T) \in X \times X^{(<\omega)}$,

if $V \subseteq \rho s$ and $T \subseteq U \rho^{c}$, then $(s, T) \in \rho_{r}^{(<\omega)}$. 
Proof. (1) $\Longrightarrow(2)$. Define a relation $\delta \subseteq X^{(<\omega)} \times X^{(<\omega)}$ by $(G, F) \in \delta$ if and only if

$$
\left(\forall(s, T) \in X \times X^{(<\omega)}\right)\left(\left(G \subseteq \rho s \wedge T \cap F \rho^{c} \neq \emptyset\right) \Longrightarrow T \cap s \rho \neq \emptyset\right) .
$$

For any $(h, W) \in X \times X^{(<\omega)}$, if $(h, W) \in\left(\rho^{-1}\right)^{(<\omega)} \circ \delta \circ\left(\rho_{r}^{-1}\right)^{(<\omega)}$, then there exists $(G, F) \in X^{(<\omega)} \times X^{(<\omega)}$ such that

$$
(h, G) \in\left(\rho_{r}^{-1}\right)^{(<\omega)} \wedge(G, F) \in \delta \wedge(F, W) \in\left(\rho^{c}\right)^{(<\omega)},
$$

i.e., $G \subseteq h \rho^{-1}=\rho h$ and $W \subseteq F \rho^{c}$. Now we have to show that $W \subseteq h \rho$. For any $w \in W$, let $s=h$ and $T=\{w\}$. Then we have $G \subseteq \rho h=\rho s$ and $\emptyset \neq$ $T \cap F \rho^{c}=\{w\} \cap F \rho^{c}=\{w\}$. From the definition of $\delta$ and $(G, F) \in \delta$, it follows $\emptyset \neq T \cap s \rho=\{w\} \cap h \rho$ what means that $w \in h \rho$. Therefore, $(h, W) \in \rho_{r}^{(<\omega)}$. We have proven inclusion $\left(\rho^{c}\right)^{(<\omega)} \circ \delta \circ\left(\rho_{r}^{-1}\right)^{(<\omega)} \subseteq \rho_{r}^{(<\omega)}$.

For any $(h, W) \in X \times X^{(<\omega)}$, if $(h, W) \in \rho_{r}^{(<\omega)}$, then $W \subseteq h \rho$. This means for any $w \in W$ holds $(h, w) \in \rho$. Since $\rho$ is weak finitely dual quasi-conjugative, there are $u_{w} \in X$ and $V_{w} \in X^{(<\omega)}$ such that

(i) $\left(u_{w}, w\right) \in \rho^{c} \wedge V_{w} \subseteq h \rho^{-1}=\rho h$, and

(ii) for all $s \in X$ and $t \in X$,

if $\left(u_{w}, t\right) \in \rho^{c}$ and $V_{w} \subseteq s \rho^{-1}=\rho s$ then $(s, t) \in \rho$.

Let $G=\bigcup_{w \in W} V_{w}, F=\left\{u_{w}: w \in W\right\}$. Then $G \subseteq h \rho^{-1}$ and $W \subseteq F \rho^{c}$ since $w \in u_{w} \rho^{c} \subseteq F \rho^{c}$. Let $s \in X$ and $T \in X^{(<\omega)}$ be arbitrary elements such that $G \subseteq s \rho^{-1}$ and $T \cap F \rho^{c} \neq \emptyset$. Then there exist $u_{w_{0}} \in F$ and $t_{0} \in T$ such that $\left(u_{w_{0}}, t_{0}\right) \in \rho^{c}$. On the other hand, according to (ii), from $w \in W$ and $V_{w} \subseteq s \rho^{-1}$, follows $\left(s, t_{0}\right) \in \rho$. This means $T \cap s \rho \neq \emptyset$. By the definition of $\delta$, we have $(G, F) \in \delta$. Hence $(h, W) \in\left(\rho^{-1}\right)^{(<\omega)} \circ \delta \circ\left(\rho_{r}^{-1}\right)^{(<\omega)}$. So, we have proven inclusion $\rho_{r}^{(<\omega)} \subseteq\left(\rho^{-1}\right)^{(<\omega)} \circ \delta \circ\left(\rho_{r}^{-1}\right)^{(<\omega)}$.

$(2) \Longrightarrow(3)$. This implication can be verified without difficulty.

$(3) \Longrightarrow(1)$. For any $(x, y) \in \rho$, there exist $V, U \in X^{(<\omega)}$ such that

(i) $y \in U \rho^{c}$ and $V \subseteq \rho x$; and

(ii) $\left(\forall(s, T) \in X \times X^{(<\omega)}\right)\left(\left(V \subseteq \rho s \wedge T \subseteq U \rho^{c}\right) \Longrightarrow(s, T) \in \rho_{r}^{(<\omega)}\right)$.

Since $y \in U \rho^{c}$, there exists $u \in U$ such that $(u, y) \in \rho^{c}$. Let $V=\left\{v_{1}, v_{2}, \ldots, v_{m}\right\}$. Then $(u, y) \in \rho^{c}$ and $(\forall j \in \bar{m})\left(\left(x, v_{j}\right) \in \rho^{-1}\right)$, i.e., the condition (i) in Definition 2.2 is satisfied. Now we check the condition (ii) in Definition 2.2. For any $(s, t) \in X \times X$, if $(u, t) \in \rho^{c}$ and $\left(s, v_{j}\right) \in \rho^{-1}(j=1,2, \ldots, m)$, i.e., $V \subseteq \rho s$ and $\{t\} \subseteq u \rho^{c} \subseteq U \rho^{c}$, then $t \in s \rho$ by the condition (ii). Thus $\rho$ is a weak finitely dual quasi-conjugative relation.

\section{REFERENCES}

[1] G. Jiang, L. Xu, J. Cai and G. Han. Normal relations on sets and applications. Int. J. Contemp. Math. Sci., 6(15)(2011), 721-726.

[2] G. Jiang and L. Xu. Dually normal relations on sets and applications. Semigroup Forum, 85(1)(2012), 75-80.

[3] S. Luo and X. Xu. Weak finitely regular relations and applications. Semigroup forum, https://doi.org/10.1007/s00233-019-10042-z

[4] D. A. Romano. Quasi-conjugative relations on sets. Mat-Kol (Banja Luka), 19(3)(2013), $5-10$.

[5] D. A. Romano and M. Vinčić. Finitelly quasi-conjugative relations. Bull. Int. Math. Virtual Inst., 3(1)(2013), 29-34. 
[6] D. A. Romano. Two new classes of relations. In: Mateljević, Stanimirović, Marić and Svetlik (eds.) Symposium 'Mathematics and applications', (24-25 May, 2013)(pp. 3439). Faculty of Mathematics, University of Belgrade, Belgrade 2014. Available online at: http://alas.matf.bg.ac.rs/ konferencija/zbornik.html.

[7] B. M. Schein. Regular elements of the semigroup of all binary relations. Semigroup Forum, 13(1976), 95-102.

[8] M. Vinčić and D. A. Romano. Finitely bi-normal relations. Gulf J. Math., 3(3)(2015), 101105.

[9] A. Zareckii. The semigroup of binary relations. Mat. Sbornik, 61(3)(1963), 291-305 (In Rus$\operatorname{sian})$

[10] X. Q. Xu and Y. M. Liu. Relational representations of hypercontinuous lattices. In: Zhang, G.Q., Lawson, J.D., Liu, Y.M., Luo, M.K. (eds.) Domain Theory, Logic, and Computation (pp. 65--74). Kluwer Academic, Dordrecht, 2003.

(D. A. Romano) International Mathematical Virtual Institute, 6, KordunašKa Street, 78000 Banja Luka, Bosnia and Herzegovina

Email address, D. A. Romano: bato49@hotmail.com 